gerade in den Bereich kommen, wo ein Andauern dieses $p_{\mathrm{H}}$ schließlich zur Gewebezerstörung führt, wenn man voraussetzt, daß die Gewebeacidose nicht nur Entzündungsfolge, sondern auch Entzündungsursache sein kann. Tatsächlich wurde bei der Untersuchung der Hyperglykämie-Wirkung auf das Wachstung von Rattentumoren ${ }^{16}$ gefunden, daß eine andauernde Hyperglykämie von $c_{\mathrm{Gk}(\mathrm{K})}=1,45 \cdot 10^{-2}$ mol $l^{-1}$ ( $\triangleq 260 \mathrm{mg}-\%$ ) zu starken Nekrotisierungen der Tumoren und starker Gewichtsverminderung vor allem der schnell-wachsenden Tumoren führt. Diese experimentellen Befunde weisen erneut darauf hin, $\mathrm{da} ß$ Nekrotisierungen infolge langdauernder starker Übersäuerungen entstehen und darum so typisch für Krebsgeschwülste sind.

16 E. J. Ciaccio, R. N. Avison u. M. S. Glitzer, 256. Proc. Amer. Ass. Cancer Res. 6, 11 [1965].
Auf der Basis der hier gewonnenen und im einzelnen besprochenen Erkenntnisse zur „Krebs-Mehrschritt-Chemotherapie" hoffen wir, durch die Kombination von verstärkter Krebsgewebe-Übersäuerung infolge zeitweiliger Blut-Glucosespiegel-Erhöhung, Extrem-Hyperthermie und Chemotherapie eine signifikante Selektivitäts-Steigerung gegenüber einem der drei Schritte allein zu erreichen. Schließlich wollen wir durch die hier dargelegten Zusammenhänge dazu beitragen, daß der Angriffspunkt KrebsgewebeUbersäuerung stärker in das Blickfeld der pharmakologischen Forschung rückt.

Die vorstehenden Untersuchungen erfolgten im Rahmen eines Auftrages des $\mathrm{Sta}$ ats sekretariats für Forschung und Technik der DDR, Berlin.

\title{
Vergleichende Untersuchungen löslicher Proteine aus Embryonalleber, Normalleber erwachsener Tiere und Transplantations-Tumoren der Ratte mit Hilfe der Agar- und Papierelektrophorese
}

\author{
W. FrITZ \\ Forschungsstelle für Experimentelle Onkologie der Deutschen Akademie der Wissenschaften \\ zu Berlin in Potsdam-Rehbrücke (Direktor: Dr. med. habil. F. Sснміdт) \\ (Z. Naturforschg. 21 b, 482-487 [1966]; eingegangen am 11. Dezember 1965)

\begin{abstract}
Embryonalleber unterscheidet sich elektrophoretisch sowohl in der qualitativen als auch quantitativen Verteilung der Fraktionen von der Normalleber erwachsener Ratten.

Als schneller wachsendes Gewebe ähnelt ihr Elektropherogramm den Transplantations-Tumoren der Ratte, besonders aber dem Hepatom.

Da Tumoren charakteristische Elektropherogramme zeigen, wird vorgeschlagen, Tumoren neben dem histologischen Bild auch elektrophoretisch zu charakterisieren. An Metastasen-freien Lebern Tumor-tragender Ratten fanden wir im Gegensatz zu anderen Autoren lediglich quantitative Veränderungen.
\end{abstract}

In früheren Untersuchungen ${ }^{1}$ hatten wir festgestellt, daß die löslichen Proteine von Gewebsextrakten aus Rattenhepatom Berlin und Horning-NierenCa des Goldhamsters im Vergleich mit den entsprechenden Normalgeweben deutliche quantitative Veränderungen zeigten. Während die schnell wandernden Proteine im Präalbumin- und Albuminbereich vermehrt waren, zeigten sich die Proteine im $\gamma$-Globulinbereich vermindert. Besonders bemerkenswert schien uns die Feststellung, daß sich bei diesen beiden Tumoren zweier verschiedener Tierarten und abweichender Organherkunft durch das Fehlen einer Fraktion im $\gamma$-Globulinbereich ein in gewisser Hin-

1 W. Fritz, Arch. Geschwulstforsch. 26, 2, 69 [1965]. sicht qualitativer Unterschied zu den entsprechenden Normalgeweben ergab.

Da Tumoren schnell wachsende Gewebe darstellen, legten wir uns die Frage vor, wie sich andere schnell wachsende Gewebe, z. B. Embryonalleber der Ratte im Vergleich zu Normalleber erwachsener Tiere, Hepatom und anderen Transplantations-Tumoren der Ratte verhalten.

\section{Material und Methodik}

Die Methodik, insbesondere auch die Elektrophoresetechnik und Auswertung wurde bereits früher beschrieben ${ }^{1}$. Die Embryonen wurden durch sectio ca. 3 Tage vor dem normalen Wurf gewonnen. Durch Dekapita- 
tion wurden sie weitgehend entblutet und die Embryonallebern mit eiskalter physiologischer Kochsalzlösung gewaschen. Die Lebern eines Wurfes wurden zusammen untersucht.

Das Gewebe aus Walker-Tumor 256, Jensen- und Yoshida-Sarkom, Guerin-Carcinom und Chlorom Shay wurde durch intramuskuläre Transplantation von Wistar-Ratten (Stamm Rehbrücke) erhalten. Die Lebern der Tumortiere wurden mit eiskalter physiologischer Kochsalzlösung in situ durch Injektion gespült. Die angewandte Auftragetechnik ${ }^{1}$ erwies sich insbesondere bei der Untersuchung der relativ kleinen Homogenatmengen aus Embryonalleber als nützlich. Die Anzahl der ausgewerteten Versuche nebst StandardAbweichung ist aus den jeweiligen Tabellen zu ersehen.

\section{Ergebnisse und Diskussion}

Bei Embryonalleber lassen sich 12 Fraktionen unterscheiden, von denen 6 anodisch und 6 kathodisch wandern (Abb. 1). Die Kennzeichnung der einzelnen Fraktionen der jeweiligen Organhomogenate

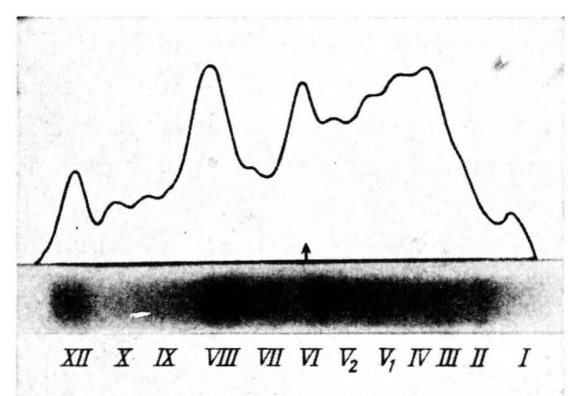

Abb. 1. Agargel-Pherogramm der Ratten-Embryonalleber.

erfolgte durch Bezifferung der Fraktionen in ihrer Reihenfolge und Zuordnung entsprechend der Wanderungsgeschwindigkeit des auf der gleichen Platte aufgetrennten Serums des jeweiligen Tieres. Während die Fraktionen I, II und III anodisch vor dem Serumalbumin liegen und damit Präalbumin-Fraktionen zugeordnet werden können, entspricht die weitere Numerierung den korrespondierenden Proteinkomponenten des Serums. Die kathodisch laufenden Fraktionen VI und VII entsprechen der $\beta_{1}$, die Fraktion VIII der $\beta_{2}$-Fraktion des Serums, die $\gamma$-Fraktionen IX, X und XI dem $\gamma$-Globulinanteil des Serums, während die post- $\gamma$-Globuline XII und XIII noch weiter kathodisch liegen. Sie fehlen im Serum und sind somit eindeutig spezifisch für Organe.

\begin{tabular}{|c|c|c|c|}
\hline & $\begin{array}{c}\text { Embryonal- } \\
\text { leber }\end{array}$ & N-Leber & $\begin{array}{c}\text { Hepatom } \\
\text { Berlin }\end{array}$ \\
& $\bar{x} \pm s$ & $\bar{x} \pm s$ & $\bar{x} \pm s$ \\
& $n=18$ & $n=14$ & $n=10$ \\
\hline I & $2,3 \pm 0,2$ & $0,9 \pm 0,1$ & $0,9 \pm 0,1$ \\
II & $4,0 \pm 0,6$ & $1,9 \pm 0,1$ & $1,3 \pm 0,2$ \\
III & $11,7 \pm 2,1$ & $5,3 \pm 0,8$ & $19,9 \pm 2,2$ \\
IV & $9,0 \pm 1,1$ & $10,6 \pm 1,3$ & $19,4 \pm 2,1$ \\
Va & $9,8 \pm 0,9$ & $15,2 \pm 1,3$ & $12,5 \pm 1,9$ \\
Vb & $8,2 \pm 0,8$ & & \\
VI & $12,7 \pm 1,5$ & $19,3 \pm 1,9$ & $12,5 \pm 1,8$ \\
VII & $7,2 \pm 0,8$ & $5,5 \pm 0,8$ & $3,1 \pm 0,2$ \\
VIII & $16,6 \pm 2,0$ & $14,0 \pm 1,0$ & $7,8 \pm 1,3$ \\
IX & $6,2 \pm 0,9$ & $6,8 \pm 1,0$ & $9,5 \pm 1,4$ \\
X & $3,2 \pm 0,6$ & $5,5 \pm 0,9$ & $4,5 \pm 1,0$ \\
XI & $-\overline{1}$ & $5,0 \pm 0,8$ & \\
XII & $8,5 \pm 1,0$ & $6,0 \pm 0,7$ & $5,9 \pm 0,9$ \\
XIII & - & $3,8 \pm 0,4$ & $0,7 \pm 0,2$ \\
\hline
\end{tabular}

Tab. 1. Prozentuale Verteilung der Eiweißfraktionen bei der agarelektrophoretischen Auftrennung von Ratten-Embryonalleber, Normalleber erwachsener Tiere und Hepatom. $\bar{x}$ arithmetisches Mittel, $s$ Standard-Abweichung, $n$ Anzahl der ausgewerteten Versuche.

Während bei Normalleber erwachsener Ratten die kathodischen Proteine, bei Hepatom dagegen die anodischen Proteine überwiegen, nehmen die Pherogramme aus Embryonalleber eine gewisse Mittelstellung ein (Abb. 1 und Tab. 1). Die schnell laufenden Fraktionen sind hier gegenüber der Normalleber ähnlich wie bei Hepatom vermehrt, besonders aber die drei ersten Fraktionen im Präalbuminbereich. Die $\alpha$-Globuline sind dagegen besser differenziert, im $\alpha_{2}$-Bereich erscheint zusätzlich eine Fraktion ausgebildet, die sowohl bei Normalleber als auch bei Hepatom fehlt. Während bei Hepatom die Fraktion III in ähnlicher Deutlichkeit wie die Albuminfraktion des Serums abgesetzt erscheint, hebt sich bei Embryonalleber keine schnell laufende Fraktion so scharf ab. Die Ähnlichkeit des Pherogramms der Embryonalleber mit dem Hepatom ist aber im Bereich der bei Krebsgeweben interessanten kathodischen Fraktionen unverkennbar. Im Bereich der $\gamma$-Globuline (Fraktionen IX, X) sind nicht wie bei Normalleber drei, sondern nur zwei Fraktionen wie bei Hepatom ausgebildet. Auch die post- $\gamma$-Globuline bestehen nicht wie bei Normalleber aus zwei, sondern nur aus einer Fraktion.

Auch andere Transplantations-Tumoren der Ratte zeigen im kathodischen Bereich ähnliche Pherogramme. Sowohl bei Walker-, Jensen-, Guerin- und Yoshida-Tumoren ist wie bei der Embryonalleber im post- $\gamma$-Globulinbereich nur eine Fraktion nachweisbar (Abbn. 2-4) ; diese Fraktion entspricht den 


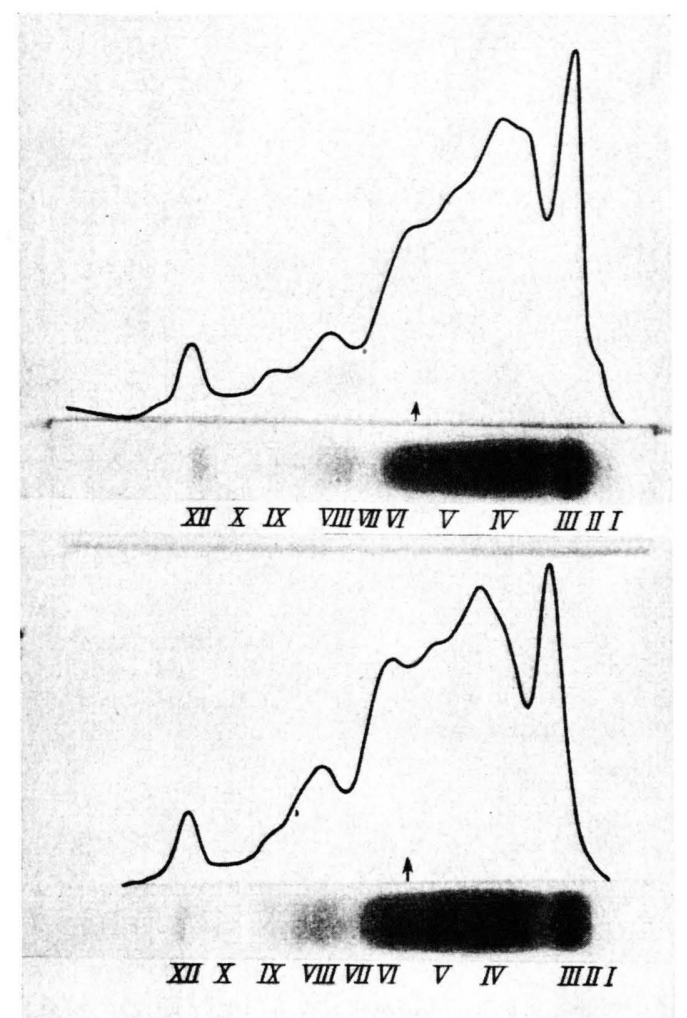

Abb. 2. Agargel-Pherogramm Walker-Tumor 256 unten, Agargel-Pherogramm Jensen-Sarkom oben.



Abb. 3. Agargel-Pherogramm Yoshida-Sarkom.

kathodisch hinter der $\gamma$-Fraktion des Serums liegenden und den somit eindeutig organspezifischen Proteinen. Weiterhin lassen sich bei Walker- und JensenTumor wie bei Hepatom und Embryonalleber im $\gamma$-Bereich nur 2 Fraktionen mit Amidoschwarz 10B anfärben gegenüber drei Fraktionen bei Normalleber (Tab. 2).

Ganz allgemein kann man sagen, daß bei allen Tumoren die schnell wandernden anodischen Fraktionen stark vermehrt sind. Dies konnten wir auch papierelektrophoretisch bestätigen. Wir befinden uns

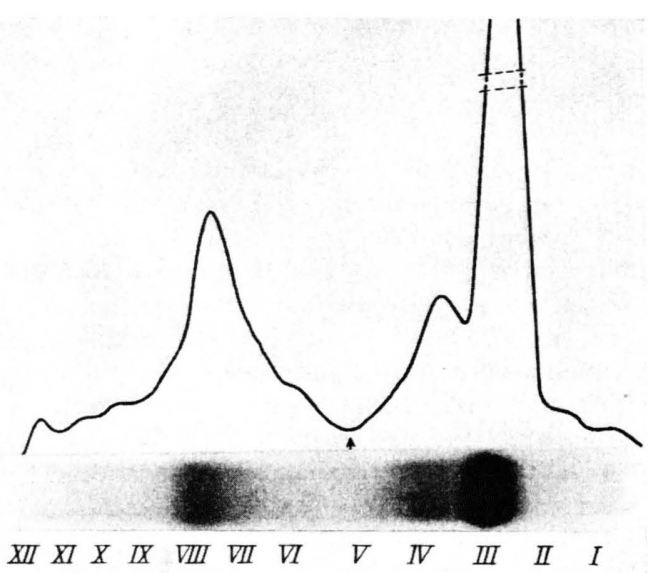

Abb. 4. Agargel-Pherogramm Guerin-Carcinom.

hier in Übereinstimmung mit nahezu allen Bearbeitern (s. l. c. ${ }^{1}$ ), während unsere Ergebnisse in gewisser Hinsicht von den Befunden von Demling ${ }^{2}$ abweichen. Er fand bei seinen papierelektrophoretisch untersuchten Neoplasmen zwar ebenso wie wir eine relativ starke Albuminbande, aber nur einen geringen Anteil an $\alpha$-Globulin, während bei ihm die mit dem $\gamma$-Globulin korrespondierende Fraktion den Hauptanteil ausmacht. Wir finden papierelektrophoretisch gerade bei den Walker- und Jensen-Tumoren die stärkste Fraktion im $\alpha$-Globulinbereich (50 und $48 \%$ ), während die Proteine im Vergleich zu allen untersuchten Transplantations-Tumoren im $\gamma$-Bereich den kleinsten Anteil ausmachen (9,1 und $8,2 \%$, bei Demling dagegen 42,6 und 42,4\%) (Abb. 6 und Tab. 3).

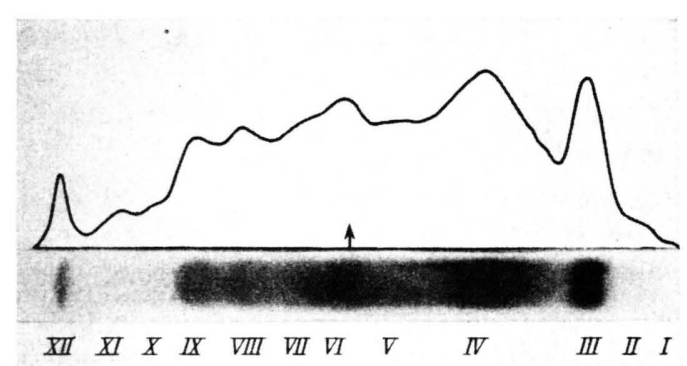

Abb. 5. Agargel-Pherogramm Chlorom-Shay.

Diese papierelektrophoretisch erzielten Ergebnisse konnten wir auch auf Agargel bestätigen, das bekanntlich eine verbesserte Auflösung ergibt. Besonders die bei der Papierelektrophorese durch die

2 L. Demling, Ärztl. Forsch. 8, I, 157 [1954]. 


\begin{tabular}{|c|c|c|c|c|c|}
\hline & $\begin{array}{c}\text { WALKER-Tumor } 256 \\
\bar{x} \pm s \\
n=14\end{array}$ & $\begin{array}{c}\text { JENSEN-Sarkom } \\
\bar{x} \pm s \\
n=12\end{array}$ & $\begin{array}{l}\text { Yoshida-Sarkom } \\
\begin{array}{c}\bar{x} \pm s \\
n=12\end{array}\end{array}$ & $\begin{array}{c}\text { GUERIN-Carcinom } \\
\begin{array}{c}\bar{x} \pm s \\
n=8\end{array}\end{array}$ & $\begin{array}{l}\text { Chlorom Shay } \\
\quad \bar{x} \pm s \\
n=14\end{array}$ \\
\hline $\begin{array}{r}\text { I } \\
\text { II } \\
\text { III } \\
\text { IV } \\
\text { V } \\
\text { VI } \\
\text { VII } \\
\text { VIII } \\
\text { IX } \\
\text { X } \\
\text { XI } \\
\text { XII } \\
\text { XIII }\end{array}$ & $\begin{array}{r}1,3 \pm 0,1 \\
1,9 \pm 0,2 \\
20,3 \pm 2,3 \\
27,9 \pm 2,5 \\
15,6 \pm 1,3 \\
16,5 \pm 1,2 \\
2,0 \pm 0,2 \\
5,7 \pm 0,3 \\
4,3 \pm 0,3 \\
2,1 \pm 0,1 \\
- \\
3,3 \pm 0,1 \\
-\end{array}$ & $\begin{array}{r}1,5 \pm 0,1 \\
2,9 \pm 0,3 \\
19,6 \pm 2,1 \\
30,6 \pm 2,5 \\
11,9 \pm 1,4 \\
16,5 \pm 1,5 \\
2,2 \pm 0,1 \\
5,9 \pm 0,4 \\
3,2 \pm 0,2 \\
1,3 \pm 0,1 \\
4,4 \pm 0,1 \\
-\end{array}$ & $\begin{array}{r}0,8 \pm 0,1 \\
1,0 \pm 0,1 \\
14,9 \pm 0,5 \\
25,9 \pm 1,1 \\
13,3 \pm 0,7 \\
14,1 \pm 0,6 \\
5,4 \pm 0,4 \\
9,4 \pm 0,6 \\
8,9 \pm 0,8 \\
1,9 \pm 0,3 \\
2,1 \pm 0,2 \\
3,7 \pm 0,3 \\
-\end{array}$ & $\begin{array}{r}1,6 \pm 0,1 \\
3,0 \pm 0,3 \\
33,6 \pm 3,1 \\
12,0 \pm 0,5 \\
5,4 \pm 0,3 \\
4,9 \pm 0,2 \\
5,6 \pm 0,4 \\
24,6 \pm 2,8 \\
3,1 \pm 0,1 \\
2,9 \pm 0,1 \\
2,1 \pm 0,1 \\
1,9 \pm 0,1 \\
-\end{array}$ & $\begin{array}{r}0,9 \pm 0,1 \\
1,9 \pm 0,2 \\
14,3 \pm 1,2 \\
19,2 \pm 1,8 \\
16,5 \pm 1,5 \\
13,8 \pm 0,7 \\
4,7 \pm 0,3 \\
10,6 \pm 0,8 \\
8,1 \pm 0,5 \\
4,1 \pm 0,4 \\
1,5 \pm 0,2 \\
4,7 \pm 0,4 \\
0,6 \pm 0,1\end{array}$ \\
\hline
\end{tabular}

Tab. 2. Prozentuale Verteilung der Eiweißfraktionen bei der agarelektrophoretischen Auftrennung von Transplantations-Tumoren der Ratte. $\bar{x}$ arithmetisches Mittel, $s$ Standard-Abweichung, $n$ Anzahl der ausgewerteten Versuche.

\begin{tabular}{|c|c|c|c|c|c|}
\hline & $\begin{array}{l}\text { WALKER- } \\
\text { Tumor } 256 \\
\quad \bar{x} \pm s \\
n=20\end{array}$ & $\begin{array}{l}\text { JEnsEn- } \\
\text { Sarkom } \\
\bar{x} \pm s \\
n=20\end{array}$ & $\begin{array}{l}\text { YoshidA- } \\
\text { Sarkom } \\
\bar{x} \pm s \\
n=20\end{array}$ & $\begin{array}{c}\text { GUERIN- } \\
\text { Carcinom } \\
\bar{x} \pm s \\
n=12\end{array}$ & $\begin{array}{l}\text { Chlorom } \\
\text { Shay } \\
\bar{x} \pm s \\
n=20\end{array}$ \\
\hline $\begin{array}{l}\text { Albumin } \\
\alpha \text {-Globulin } \\
\beta \text {-Globulin } \\
\gamma \text {-Globulin }\end{array}$ & $\begin{array}{r}20,0 \pm 2,8 \\
48,3 \pm 3,4 \\
22,6 \pm 2,3 \\
9,1 \pm 1,4\end{array}$ & $\begin{array}{r}18,1 \pm 2,4 \\
50,1 \pm 3,2 \\
23,6 \pm 2,5 \\
8,2 \pm 1,2\end{array}$ & $\begin{array}{l}14,3 \pm 1,9 \\
39,1 \pm 2,7 \\
31,4 \pm 2,0 \\
15,2 \pm 2,1\end{array}$ & $\begin{array}{l}32,4 \pm 3,4 \\
12,1 \pm 1,1 \\
43,2 \pm 4,1 \\
12,3 \pm 1,1\end{array}$ & $\begin{array}{l}17,3 \pm 2,1 \\
35,1 \pm 2,3 \\
28,2 \pm 1,9 \\
19,4 \pm 1,7\end{array}$ \\
\hline
\end{tabular}

Tab. 3. Prozentuale Verteilung der Eiweißfraktionen bei der papierelektrophoretischen Auftrennung. Zuordnung nach Serumfraktionen des gleichen Tieres. $\bar{x}$ arithmetisches Mittel, $s$ Standard-Abweichung, $n$ Anzahl der ausgewerteten Versuche.

Schleppenbildung schlecht differenzierten Globuline werden bei dieser Technik gut getrennt.

Beim Vergleich der von uns auf Papier und Agar getrennten Tumorhomogenaten ergab sich bei Fraktionen entsprechender Wanderungsgeschwindigkeiten weitgehende Übereinstimmung (Tab. 2 und 3).

Die in freier Elektrophorese durch Sonof und CoHEN $^{3}$ und in unserem Arbeitskreis durch Schwenke ${ }^{4}$ aufgetrennten Walker- und Jensen-Tumoren ergaben ebenfalls einen kleinen Proteinanteil mit $\gamma$-Globulinbeweglichkeit (3,5 und 5,5\%). Der Hauptanteil schneller wandernder Proteine kann den Präalbumin-, Albumin- und $\alpha$-Globulinbereichen zugeordnet werden.

Auch $\mathrm{BARRY}^{5}$ fand in der freien Elektrophorese der löslichen Proteine durch Benzpyren und Methylcholanthren induzierten Rattenfibrosarkomen, daß die Proteine mit der Beweglichkeit der $\alpha$-Globuline den Hauptanteil mit $50-70 \%$, die $\gamma$-Globuline dagegen nur $5 \%$ ausmachten.

3 S. Sorof u. P. P. Cohen, Cancer Res. 11, 376 [1951].

4 K. D. Schwenke, Z. Krebsforsch. 68, 112 [1966].
Während bei Walker- und Jensen-Tumoren die schnell wandernden anodischen Fraktionen stark überwiegen (2/3:1/3) (Abb. 2), sind bei Chlorom die kathodischen Fraktionen vermehrt und auch sehr

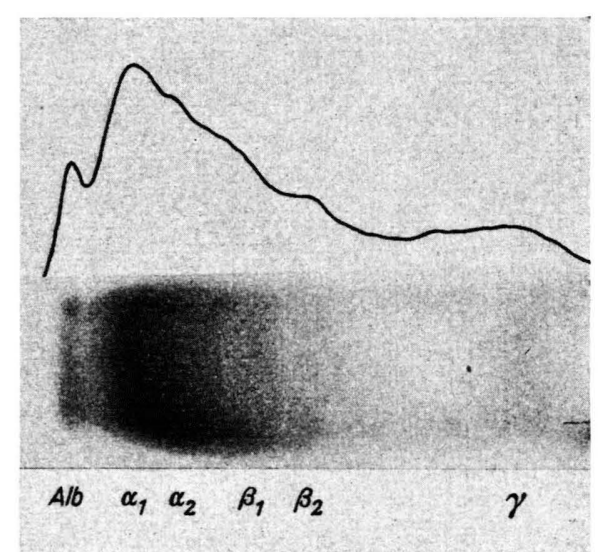

Abb. 6. Papier-Elektropherogramm Walker-Tumor 256.

5 G. T. BARRY, Cancer Res. 10, 694 [1950]. 
gut getrennt (Abb. 5). Das prozentuale Verhältnis der anodischen zu den kathodischen Fraktionen ist nahezu ausgeglichen $(52: 48)$. Beim Guerin-Carcinom sind die Fraktionen IV, V und VI, die bei anderen Tumoren den Hauptanteil der Proteine ausmachen, nur schwach ausgebildet (Abb. 4). Das wird besonders im Papier-Elektropherogramm deutlich.

Da jeder Tumor ein charakteristisches Elektropherogramm besitzt, dürfte es nützlich sein, neben der routinemäßig betriebenen Serumelektrophorese auch Tumorhomogenate trägerelektrophoretisch entweder auf Agar oder auch auf Acetylcellulose ${ }^{6}$ aufzutrennen. Mit der von uns beschriebenen Auftragetechnik auf $\operatorname{Agar}^{1}$ ist es möglich, kleinste Mengen, z. B. auch bioptisch gewonnenes Tumormaterial des Menschen zu untersuchen. Somit könnte man einen Tumor neben seinem histologischen Bild auch elektrophoretisch charakterisieren.

Während die Ratten-Embryonalleber als schnell wachsendes Gewebe sich auch elektrophoretisch von der Leber erwachsener Tiere deutlich unterscheidet - auch Nixolov ${ }^{7}$ diskutiert eine Ähnlichkeit des Pherogramms mit dem des transplantablen Hepatoms - gilt das für ein anderes schnell wachsendes Gewebe, die teilhepatektomierte Leber offensichtlich nicht. Sorof und Cohen ${ }^{3,8}$ haben in der freien Elektrophorese keine signifikante quantitative Abweichung der langsam wandernden sogenannten h-Kom- ponenten in regenerierenden Rattenlebern 12 Std., 1, 2, 4, 6 und 8 Tage nach partieller Hepatektomie des Hauptleberlappens gefunden.

Zu ähnlichen Ergebnissen kam auch DeLamirande ${ }^{9}$. Ebenfalls mit der Methode der freien Elektrophorese fand er zwar zunächst eine Verminderung der langsamen h-Proteine, dann aber eine Normalisierung auf den normalen Wert. Auch papierelektrophoretisch stellte Guidotтi ${ }^{10}$ bei regenerierender Rattenleber eine weitgehende Übereinstimmung des elektrophoretischen Bildes wie bei der Restleber fest. Lediglich 24 Stdn. nach der partiellen Hepatektomie war der Albumingipfel erhöht.

Was das elektrophoretische Verhalten der Lebern Tumor-tragender Tiere betrifft, wurde schon in den ersten Elektrophoresearbeiten von Sorof und CoHeN $^{3}$ die Beeinflussung eines Lebertumors auf das umgebende Lebergewebe untersucht, ohne daß qualitative Veränderungen gefunden werden konnten. Aus unserem Arbeitskreis konnte $\mathrm{ScHwenke}^{4}$ mit der Methode der freien Elektrophorese eine geringe Erhöhung der $\alpha_{1}$ - und $\alpha_{2}$-Globuline und eine Erniedrigung der h-Proteine feststellen. GorJukhina ${ }^{11}$ aus dem Arbeitskreis von PARshin ${ }^{12}$ fand jedoch sowohl bei der Papier- als auch Stärkeelektrophorese bei Lebern Tumor-tragender Tiere eine größere Anzahl Fraktionen als in der Normalleber. Wir haben aber an allen von uns untersuchten Metastasen-freien

\begin{tabular}{|c|c|c|c|c|c|c|c|c|}
\hline & $\begin{array}{l}\text { Normal- } \\
\text { leber } \\
\bar{x} \pm s \\
n=14\end{array}$ & $\begin{array}{l}\text { Ascites- } \\
\text { leber } \\
\bar{x} \pm s \\
n=18\end{array}$ & $\begin{array}{c}\text { WALKER- } \\
\text { leber } \\
\bar{x} \pm s \\
n=9\end{array}$ & $\begin{array}{c}\text { JENSEN- } \\
\text { leber } \\
\bar{x} \pm s \\
n=6\end{array}$ & $\begin{array}{l}\text { Hepatom- } \\
\quad \text { leber } \\
\quad \bar{x} \pm s \\
n=6\end{array}$ & $\begin{array}{c}\text { YoshidA- } \\
\text { leber } \\
\bar{x} \pm s \\
n=6\end{array}$ & $\begin{array}{c}\text { Chlorom- } \\
\text { leber } \\
\bar{x} \pm s \\
n=6\end{array}$ & $\begin{array}{c}\text { Guerin- } \\
\text { leber } \\
\bar{x} \pm s \\
n=6\end{array}$ \\
\hline II & $2,8 \pm 0,1$ & $2,8 \pm 0,2$ & $2,5 \pm 0,2$ & $2,0 \pm 0,3$ & $2,4 \pm 0,2$ & $2,6 \pm 0,2$ & $2,6 \pm 0,2$ & $1,9 \pm 0,1$ \\
\hline III & $5,3 \pm 0,8$ & $5,8 \pm 0,9$ & $4,6 \pm 0,5$ & $5,3 \pm 0,7$ & $7,6 \pm 0,8$ & $6,2 \pm 0,5$ & $6,1 \pm 0,4$ & $6,3 \pm 0,6$ \\
\hline V & $25,8 \pm 1,3$ & $31,9 \pm 2,1$ & $32,8 \pm 2,3$ & $32,6 \pm 2,2$ & $32,6 \pm 2,2$ & $33,5 \pm 2,1$ & $33,6 \pm 2,5$ & $36,4 \pm 2,3$ \\
\hline $\begin{array}{r}\text { VI } \\
\text { VII }\end{array}$ & $24,8 \pm 1,9$ & $18,4 \pm 1,7$ & $21,4 \pm 1,8$ & $21,8 \pm 1,9$ & $20,3 \pm 1,8$ & $21,5 \pm 1,6$ & $19,1 \pm 1,7$ & $15,2 \pm 1,5$ \\
\hline $\begin{array}{l}\text { VIII } \\
\text { IX }\end{array}$ & $14,0 \pm 1,0$ & $15,5 \pm 1,2$ & $14,5 \pm 1,1$ & $14,3 \pm 1,0$ & $15,0 \pm 1,0$ & $13,5 \pm 0,9$ & $14,0 \pm 1,0$ & $13,0 \pm 0,9$ \\
\hline XI & $17,7 \pm 1,0$ & $13,7 \pm 0,9$ & $12,2 \pm 0,8$ & $12,6 \pm 0,7$ & $14,1 \pm 1,0$ & $12,5 \pm 0,9$ & $17,5 \pm 1,1$ & $18,0 \pm 1,3$ \\
\hline $\begin{array}{r}\text { XII } \\
\text { XIII }\end{array}$ & $9,8 \pm 0,7$ & $17,7 \pm 1,6$ & $12,1 \pm 1,5$ & $12,5 \pm 1,4$ & $9,6 \pm 0,8$ & $13,0 \pm 0,9$ & $11,1 \pm 1,2$ & $10,3 \pm 1,2$ \\
\hline
\end{tabular}

Tab. 4. Prozentuale Verteilung der Eiweißfraktionen bei der agarelektrophoretischen Auftrennung von Lebern Tumor-tragender Ratten. $\bar{x}$ arithmetisches Mittel, $s$ Standard-Abweichung, $n$ Anzahl der ausgewerteten Versuche.

${ }^{6}$ E. Afonso, J. clin. Pathol. 16, 375 [1963].

7 T. K. Nikolov u. A. A. Hadjiolov, Biochimia 26, 523 [1961].

8 S. Sorof, B. Claus u. P. P. Cohen, Cancer Res. 11, 873 [1951].

9 Delamirande, Cancer 6, 179 [1953].
10 G. Guidotti u. E. Clerici, Experientia [Basel] 14, 341 [1958].

11 T. A. Gorjukhina, Vopr. Onkol. 5, 44 [1964].

12 A. N. Parshin, T. A. Gorjukhina, E. V. Smirnova, V. S. Misheneva u. M. N. Tagmisjan, Unio intern. contra cancrum Acta 20, II, 955 [1964]. 
Tumorlebern von Ratten mit Transplantations-Tumoren keine qualitativen Veränderungen durch $\mathrm{Pa}$ pier- und Agarelektrophorese feststellen können. Als quantitative Veränderungen bei den Lebern Tumortragender Ratten ergab sich eine Erhöhung der $\alpha$ Globuline um $6-8 \%$, die Präalbumin- und $\beta_{2}$-Fraktion blieben unverändert, während die $\beta_{1}$-Fraktion um $3-6 \%$ und die $\gamma$-Fraktion um $3-5 \%$ vermindert war. Die organspezifischen Proteine der post- $\gamma$ Globulinfraktion waren bei den Lebern der verschiedensten Transplantations-Tumoren nur leicht erhöht, während sie bei Ascites-Hepatom Paris einen um $7 \%$ größeren Betrag aufwiesen (Tab. 4). 